\title{
Fasudil inhibits prostate cancer-induced angiogenesis in vitro
}

\author{
WEIHUA CHEN ${ }^{1,2 *}$, KAILI MAO ${ }^{2 *}$, THONG HUA-HUY $^{1}$, YIHUA BEI $^{1}$, \\ ZHONGMIN LIU $^{3}$ and ANH-TUAN DINH-XUAN ${ }^{1,3}$
}

\author{
${ }^{1}$ Medical School, Service de Physiologie, Paris Descartes University, EA 2511, Hôpital Cochin, 75014 Paris, France; \\ ${ }^{2}$ Department of Urology, Shanghai East Hospital, Tongji University, School of Medicine, Shanghai 200120; ${ }^{3}$ Clinical and \\ Translational Research Center, Shanghai East Hospital, Tongji University, School of Medicine, Shanghai 200120, P.R. China
}

Received June 16, 2014; Accepted August 19, 2014

DOI: $10.3892 /$ or.2014.3491

\begin{abstract}
Inhibition of angiogenesis is an important therapeutic strategy for advanced stage prostate cancer ( $\mathrm{PCa}$ ). RhoA/Rho-associated protein kinases (ROCK) are key regulators of the cytoskeleton and have been implicated in $\mathrm{PCa}$ angiogenesis. We investigated the anti-angiogenic effects of fasudil, a ROCK inhibitor, on PCa-induced angiogenesis in vitro. Proliferation of PCa-conditioned human umbilical vein endothelial cells (HUVECs) was assessed using a bromodeoxyuridine (BrdU) assay, and migration was assessed with a wound healing assay. In vitro angiogenesis of $\mathrm{PCa}$-conditioned HUVECs was evaluated by tube formation and a spheroid sprouting assay. Fasudil inhibited PCa-induced endothelial cell proliferation at a concentration of $100 \mu \mathrm{M}$, and also decreased PCa-induced endothelial cell migration at a concentration of $30 \mu \mathrm{M}$. In the in vitro angiogenesis assay, fasudil exerted a more significant effect. Tube formation was significantly inhibited at fasudil concentrations exceeding $3 \mu \mathrm{M}$, and spheroid sprouts were significantly thinner and shorter (at fasudil concentrations of 10 and $30 \mu \mathrm{M}$, respectively). Western blotting results showed that expression of phosphorylated myosin phosphatase target subunit 1 (MYPT-1) was significantly lower after fasudil treatment, confirming that fasudil inhibited ROCK activity in these model systems. These data suggest that fasudil may be a useful anti-angiogenic agent for PCa.
\end{abstract}

\section{Introduction}

Prostate cancer ( $\mathrm{PCa}$ ) is the most common malignancy and the second leading cause of cancer-related death in men in

Correspondence to: Professor Anh-Tuan Dinh-Xuan, Service de Physiologie - Explorations Fonctionnelles, Hôpital Cochin, Université Paris Descartes, 27 Rue du Faubourg Saint-Jacques, 75014 Paris, France E-mail: anh-tuan.dinh-xuan@cch.aphp.fr

Professor Zhongmin Liu, Clinical and Translational Research Center, Shanghai East Hospital, Tongji University, School of Medicine, 150 Jimo Road, Shanghai 200120, P.R. China

E-mail: zhongmin_liu@sina.com

${ }^{*}$ Contributed equally

Key words: fasudil, ROCK, angiogenesis, prostate cancer
Western countries. Advanced and metastatic stages of the disease are found in 35\% of patients with PCa diagnosed at autopsy (1). Among patients with localized cancer who are eligible for radical prostatectomy, $\sim 35 \%$ will develop recurrence (metastatic disease) within 10 years of surgery $(2,3)$.

Androgen deprivation therapy (ADT) can be effective in patients who present with or progress to advanced or metastatic disease. Unfortunately, the median duration of response to ADT is limited to between 8 months and 3 years (4), and these patients will eventually become castration resistant. Chemotherapy is an effective treatment for castration-resistant $\mathrm{PCa}$, but the median duration of response is only 10.3 months (5). There is clearly an urgent need to develop additional systemic interventions for patients with progressive $\mathrm{PCa}$. Angiogenesis plays a crucial role in $\mathrm{PCa}$ progression and metastasis. Microvessel density (MVD) has been found to be more prominent in $\mathrm{PCa}$ than in benign prostatic hyperplasia (BPH) and normal tissue $(6,7)$. It has been reported that MVD increases with increased Gleason's score, particularly in poorly differentiated PCa (8). MVD was also significantly correlated with cancer-specific survival in 221 patients with PCa followed up for a median of 15 years (9).

Vascular endothelial growth factor (VEGF) is the most prominent regulator of physiological angiogenesis and has been correlated with increased levels of angiogenesis in clinical studies comparing PCa with BPH (7). Higher VEGF expression and serum levels have also been found in patients with metastasis or poorly differentiated tumors, as well as in those with a poor prognosis (10-13). However, it has become increasingly apparent that current anti-angiogenic therapy targeting VEGF has only a modest effect in the clinical setting.

RhoA and its downstream effector, Rho-associated protein kinase (ROCK), serve as key regulators of extracellular stimulus-mediated signaling networks that are involved in various cellular processes, including motility, mitosis, proliferation and apoptosis (14). Suppression of the RhoA/ROCK signaling pathway with the ROCK inhibitor, Y-27632, was found to inhibit VEGF-induced angiogenesis in vitro (15). Another ROCK inhibitor, fasudil, has been shown to inhibit VEGF-induced angiogenesis in vitro and in vivo (16). A study carried out on endothelial cells from transgenic adenocarcinoma of the mouse prostate (TRAMP) mice revealed that their behavior correlated with a constitutively high level of baseline activity of Rho GTPase and ROCK (17). This suggests that the 
RhoA/ROCK pathway has an important role in PCa angiogenesis. However, the anti-angiogenic effects of ROCK inhibitors in PCa are unclear. We investigated the role of fadusil, a ROCK inhibitor, that has been approved for clinical use for pulmonary arterial hypertension, on PCa-induced angiogenesis in vitro.

\section{Materials and methods}

Cell culture. Human umbilical vein endothelial cells (HUVECs) were purchased from PromoCell (C-12200; Heidelberg, Germany) and cultured in endothelial cell growth medium (C-22010; PromoCell). Cultures were maintained at $37^{\circ} \mathrm{C}$ in a humidified atmosphere containing $5 \% \mathrm{CO}_{2}$. Subcultures were obtained by trypsinization and were used for experiments at passages 3 to 9. Before performing the experiments, the cells were made quiescent by incubating overnight in endothelial cell basal medium (C-22210) containing $0.5 \%$ (w/v) fetal bovine serum (FBS). The PCa cell line, PC-3, was purchased from The European Collection of Cell Cultures and grown in F-12K (Gibco-Invitrogen, Carlsbad, CA, USA) containing $10 \%(\mathrm{w} / \mathrm{v}) \mathrm{FBS}$. PC-3 cells were seeded at a concentration of $6 \times 10^{6}$ cells/T75 flask. On the following day, the medium was replaced with basal medium without FBS, and the supernatants were harvested after a 24-h incubation to serve as conditioned medium (PC3CM). Recombinant human VEGF 165 was purchased from R\&D Systems (293VE; Minneapolis, MN, USA). HUVECs were cultured in endothelial cell basal medium plus $2 \%$ (w/v) FBS (control group), in PC3CM plus 2\% (w/v) FBS (PC3CM group), or in basal medium plus $2 \%$ (w/v) FBS and $30 \mathrm{ng} / \mathrm{ml}$ VEGF (VEGF group).

Cell proliferation assay. HUVEC proliferation was evaluated using a BrdU incorporation assay kit (Amersham; Cell Proliferation Biotrak ELISA System; RPN250; GE Healthcare, Little Chalfont, UK), according to the manufacturers' instructions. In brief, HUVECs were plated in 96-well microculture plates $\left(3 \times 10^{3}\right.$ cells/well). After a 48 -h incubation at $37^{\circ} \mathrm{C}$ in a $5 \% \mathrm{CO}_{2}$ atmosphere, with or without fasudil (1-100 $\left.\mu \mathrm{M}\right), 10 \mu \mathrm{l}$ BrdU labeling reagent was added, and the cells were cultured for a further $2 \mathrm{~h}$. Cells were washed twice with Dulbecco's PBS (D8537; Sigma-Aldrich, St. Louis, MO, USA), fixed with fixative solution and then blocked with blocking buffer. BrdU incorporation was revealed by incubation with $100 \mu \mathrm{l} /$ well horseradish peroxidase (HRP)-labeled anti-BrdU working solution for $\sim 90 \mathrm{~min}$. Tetramethylbenzidine (TMB) substrate at room temperature was added at $100 \mu \mathrm{l} /$ well for $20 \mathrm{~min}$. Absorbance was measured at $450 \mathrm{~nm}$ using a microplate reader. All determinations were performed in octuplicate, and each experiment was repeated three times.

Cell migration assay. Cell motility was assessed using a wound-healing migration assay. HUVECs were seeded to full confluency in 6-well plates. The following day, a uniform scratch was made down the centre of the well using a $100-\mu 1$ micropipette tip, and the cells were washed twice with PBS. After incubation for $24 \mathrm{~h}$ with or without $30 \mu \mathrm{M}$ fasudil in the control, PC3CM and VEGF groups, the cells were fixed and photographed. Photographic imaging was performed using a Leica inverted microscope. Cell migration was quantified by measuring the ratio of the migration area to the total area of the wound gap. Each experiment was repeated three times.

Tube formation assay. Ninety-six-well plates were chilled to $4^{\circ} \mathrm{C}$ and coated with $50 \mu \mathrm{l}$ of Matrigel (354234; BD Biosciences, Oxford, UK) per well. Freshly passaged HUVECs were seeded onto the gel. Endothelial tube morphogenesis was carried out in the presence or absence of fasudil (3-30 $\mu \mathrm{M})$. Endothelial tube formation was observed after $16 \mathrm{~h}$ and photographed under phase contrast microscopy using a Leica inverted microscope. Quantification of the digital images was performed by counting the total number of tubes in five 40x fields, and total tube length was quantified using Image $J^{\mathrm{TM}}$ software $(\mathrm{NIH}$, Bethesda, MD, USA). Tube formation was expressed as fold change or percentage, compared to the controls. All determinations were performed three times, and each experiment was repeated three times.

Spheroid sprouting assay. HUVECs were suspended in culture medium containing $0.2 \%(\mathrm{w} / \mathrm{v})$ methylcellulose (Sigma-Aldrich) and seeded in non-adherent round-bottom 96-well plates (Greiner, Frickenhausen, Germany). All suspended cells formed a single spheroid in each well of defined size and cell number ( 400 cells/spheroid). Spheroids were left to form for $24 \mathrm{~h}$ and then embedded in $1.5 \mathrm{mg} / \mathrm{ml}$ collagen gel. The spheroid-containing gel was rapidly transferred to pre-warmed 24-well plates and allowed to polymerize for $30 \mathrm{~min}$. Endothelial basal medium or PC3CM with or without fasudil (1-100 $\mu \mathrm{M})$ was then added to the surface of the gel $(500 \mu \mathrm{l} /$ well). After $16 \mathrm{~h}$, images were captured using a Leica inverted microscope. Sprouting was quantified using $\mathrm{NIH}$ ImageJ software by measuring the cumulative sprout length, which consisted of every sprout from 10 spheroids in each group.

Western blot assay. Protein was extracted on ice from the cultured HUVECs with cold RIPA lysis buffer (9806; Cell Signaling Technology, Boston, MA, USA) containing Pierce ${ }^{\mathrm{TM}}$ Protease and Phosphatase Inhibitor (88669; Thermo Scientific, Rockford, IL, USA). Lysates were centrifuged at 12,000 x g for $20 \mathrm{~min}$ at $4^{\circ} \mathrm{C}$, and the supernatant was collected. Total protein concentrations were determined using a bicinchoninic acid assay (BCA) protein assay kit (23250; Thermo Scientific). Equal amounts of protein were subjected to sodium dodecyl sulfate polyacrylamide gel electrophoresis and then electrically transferred onto nitrocellulose membranes. The membranes were blocked for $1 \mathrm{~h}$ with $5 \%$ (w/v) non-fat milk in PBS-0.1\% (v/v) Tween-20 (PBST) and incubated with primary antibodies against MYPT-1 (1:1,000; sc-25618; Santa Cruz Biotechnology, Dallas, TX, USA), phospho-MYPT-1 (1:500; ABS45; Millipore, Billerica, MA, USA), anti-ROCK1 (1:500; sc-6055), anti-ROCK2 (1:1,000; sc-1851; both from Santa Cruz Biotechnology) and $\beta$-actin (1:500; ab8229; Abcam, Cambridge, UK) overnight at $4^{\circ} \mathrm{C}$. Finally, the membrane was incubated with HRP-conjugated secondary antibodies as follows: goat anti-mouse IgG-HRP (1:5,000; sc-2005; Santa Cruz Biotechnology), rabbit anti-goat IgG-HRP (1:10,000; sc-2768; Santa Cruz Biotechnology), goat anti-rabbit IgG-HRP (1:10,000; sc-2004; Santa Cruz Biotechnology) for $1 \mathrm{~h}$ at room temperature. After washing three times with PBST, proteins 
A

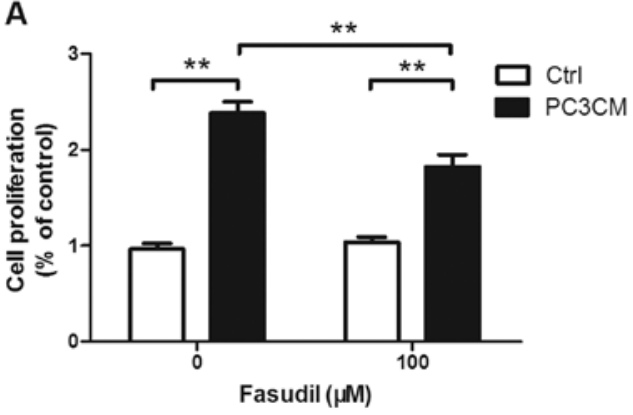

B

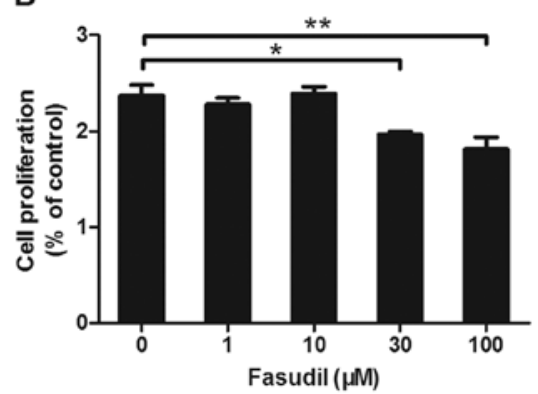

Figure 1. Effects of fasudil on PCa-induced HUVEC proliferation. HUVECs were cultured in endothelial culture medium or PC3CM for $48 \mathrm{~h}$, with or without various concentrations of fasudil. A BrdU assay was used to assess the effects of fasudil on cell proliferation. (A) Cell proliferation was significantly decreased in the PC $3 \mathrm{CM}$ group treated with $100 \mu \mathrm{M}$ fasudil (P<0.01). (B) The inhibitory effect of fasudil on PC3CM-induced HUVEC proliferation was dose-dependent. The results shown are the mean \pm SEM of three independent experiments. ${ }^{*} \mathrm{P}<0.05,{ }^{* *} \mathrm{P}<0.01$. HUVECs, human umbilical vein endothelial cells; $\mathrm{PCa}$, prostate cancer; PC3CM, PCa cell line PC3-conditioned medium.

A

$\mathrm{Oh}$

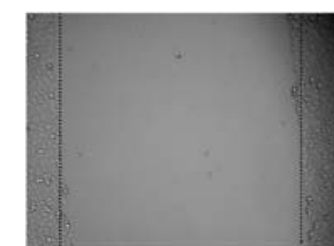

PC3CM
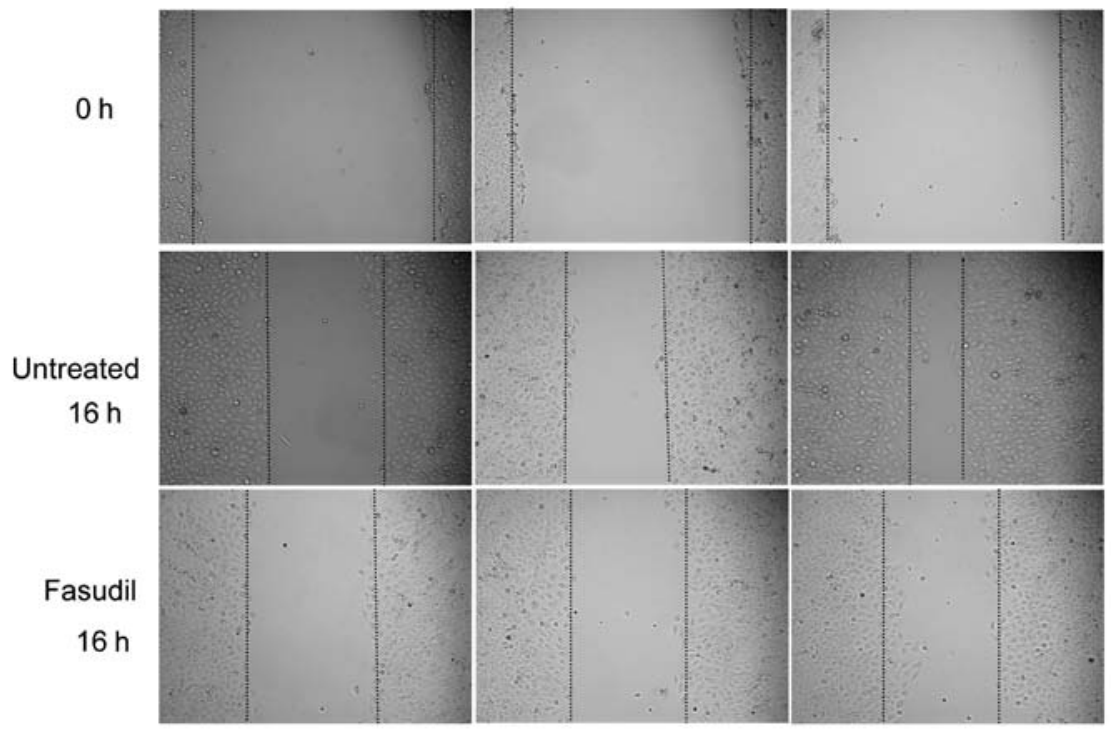

B

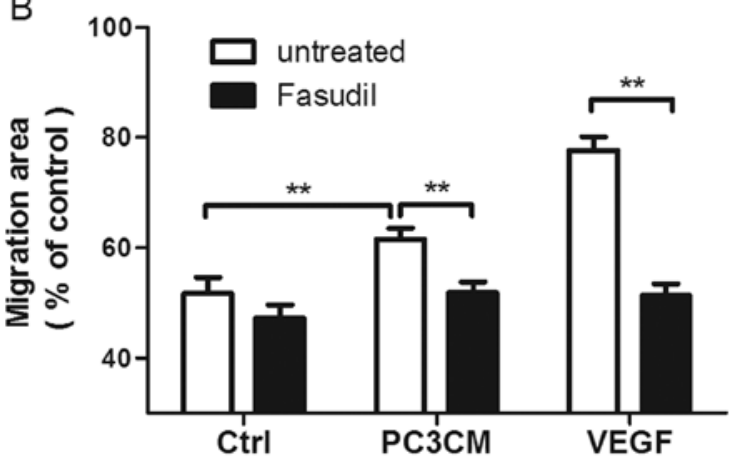

Figure 2. Fasudil inhibits PC3CM-induced HUVEC migration. (A) Fasudil (30 $\mu \mathrm{M})$ significantly retarded PC3CM-cultured HUVEC migration into the scratched gap. (B) Quantification of wound healing. HUVEC migration was quantified by counting the percentage of the migration area $16 \mathrm{~h}$ after fasudil treatment. Values are the mean \pm SEM from 4 cultures each in triplicate experiments. ${ }^{* *} \mathrm{P}<0.01$. HUVECs, human umbilical vein endothelial cells; PCa, prostate cancer; PC3CM, PCa cell line PC3-conditioned media, SEM, standard error of the mean.

were visualized using an ECL Prime Western blotting detection kit (GE Healthcare). Photographs of the protein bands were captured using a digital imaging system (ImageQuant LAS; GE Healthcare), and densitometric measurements of band intensity in the western blotting were performed using
NIH ImageJ software. The results shown are representative of three or more independent experiments.

Statistical analysis. Data are expressed as means \pm standard deviation. Significance of differences was determined by the 
A
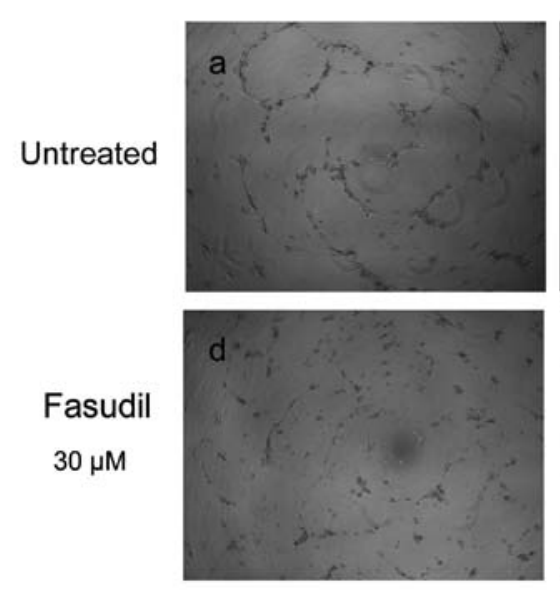

B

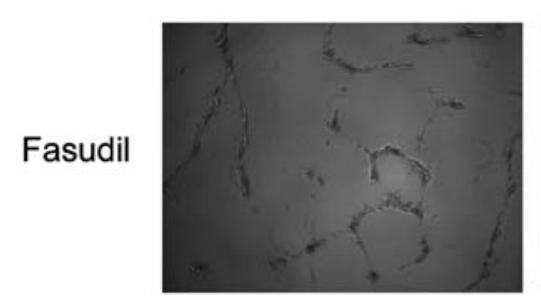

$3 \mu \mathrm{M}$

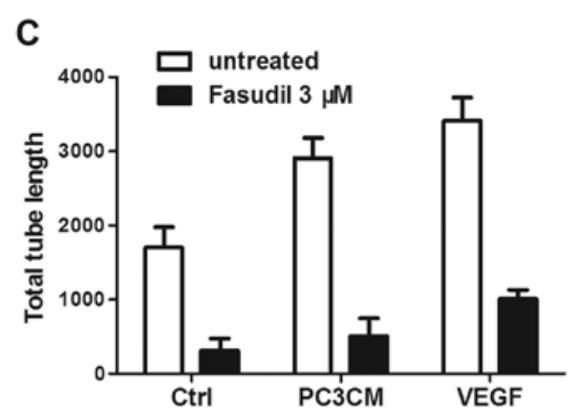

PC3CM
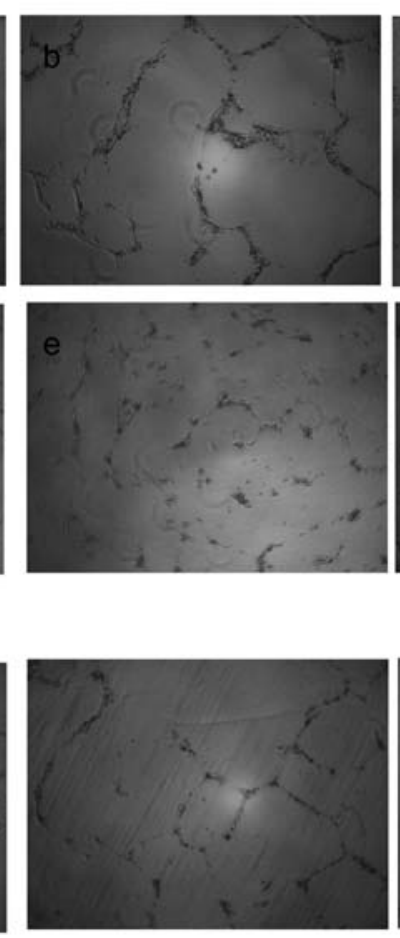

$10 \mu \mathrm{M}$
VEGF
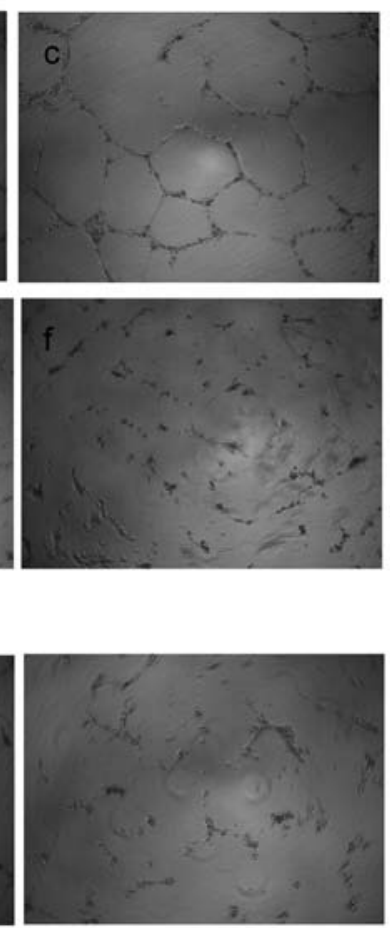

$30 \mu \mathrm{M}$

\section{D}

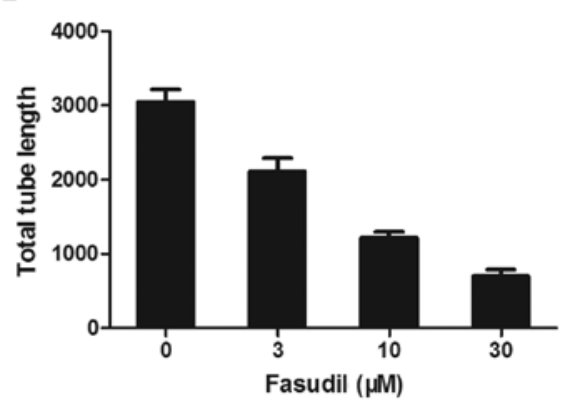

Figure 3. PC3CM-induced capillary-like tube formation is reduced by fasudil. (A) HUVECs were cultured on Matrigel with basal medium (a), PC3CM (b), or basal medium containing $30 \mathrm{ng} / \mathrm{ml}$ VEGF (c) either in the absence (a-c), or presence of $30 \mu \mathrm{M}$ fasudil (d-f). Images were captured after $16 \mathrm{~h}$ under a Leica inverted phase-contrast microscope. Representative images are shown above. PC3CM (b) increased the total tube length compared to the control (a), whereas fasudil strongly inhibited tube formation (e). (B) Dose-dependent effects of fasudil on total tube length in PC3CM-cultured HUVECs. As the fasudil concentration increased, the total tube length gradually decreased. (C and D) Total tube length was quantified by evaluating five fields in each experiment, and data were verified by three independent experiments. Values are expressed as the mean \pm SEM. HUVECs, human umbilical vein endothelial cells; PCa, prostate cancer; PC3CM, PCa cell line PC3-conditioned media; SEM, standard error of the mean; VEGF, vascular endothelial growth factor.

two-tailed Student's t-test or the analysis of variance least significant difference (ANOVA LSD) test. A P-value $<0.05$ was considered to indicate a statistically significant difference.

\section{Results}

Fasudil inhibits PC3CM-induced HUVEC proliferation. Endothelial cell proliferation is crucial for angiogenesis. PC3CM-treated HUVECs were exposed to fasudil concentrations ranging from 1 to $100 \mu \mathrm{M}$, and HUVEC proliferation was examined using a BrdU assay. Fasudil concentrations of $\geq 30 \mu \mathrm{M}$ had a significant inhibitory effect on PC3CM-induced cell proliferation, while proliferation in the control group was unchanged (Fig. 1).
Fasudil inhibits PC3CM-induced HUVEC migration. The inhibitory effects of fasudil on endothelial cell motility were assessed using a wound-healing migration assay. Fasudil $(30 \mu \mathrm{M})$ significantly decreased the number of cells migrating into the scratched gap in the control, PC3CM and VEGF groups, indicating the potent inhibitory effect of fasudil on HUVEC movement and migration. VEGF increased HUVEC migration significantly more than PC3CM-induced HUVEC migration. After treatment with $30 \mu \mathrm{M}$ fasudil, all migrations decreased to similar levels (Fig. 2).

Fasudil inhibits PC3CM-induced HUVEC tube formation. The effect of fasudil on capillary-like structure formation in vitro was examined using a 3-dimensional (3D) Matrigel 
A
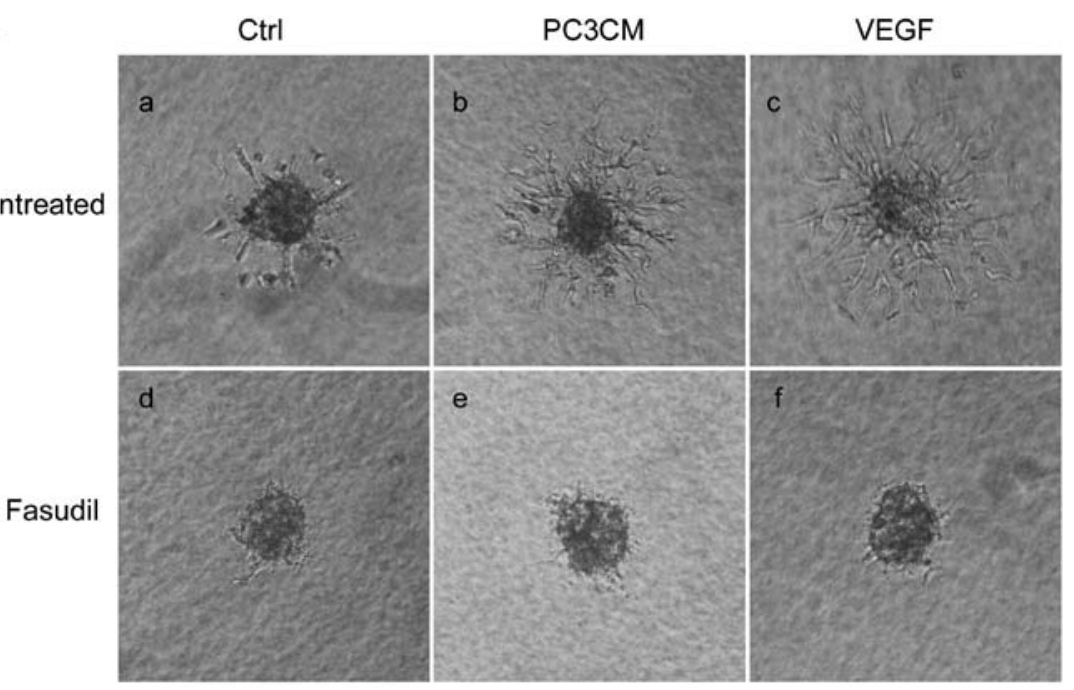

B
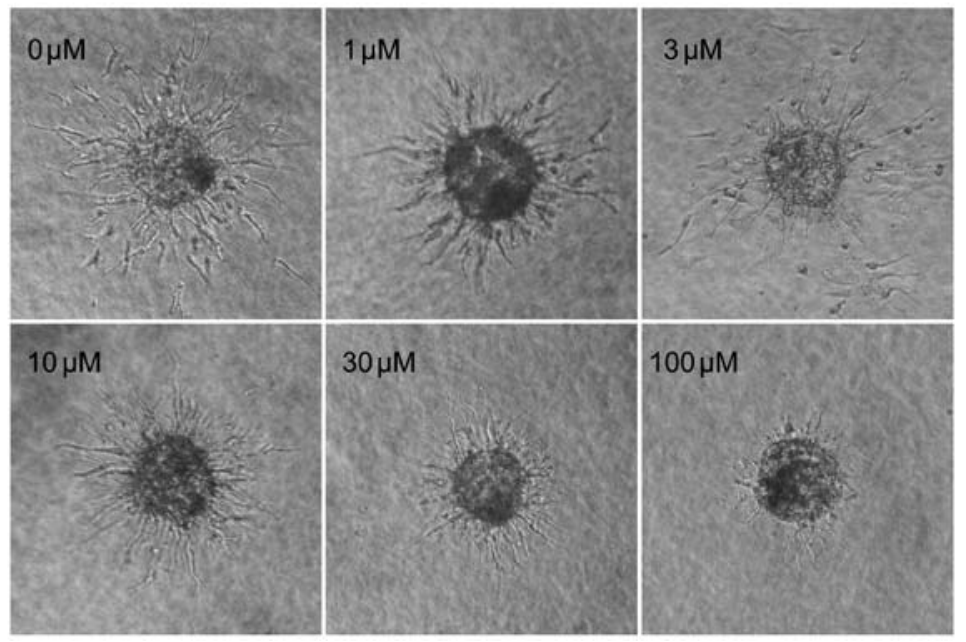

C

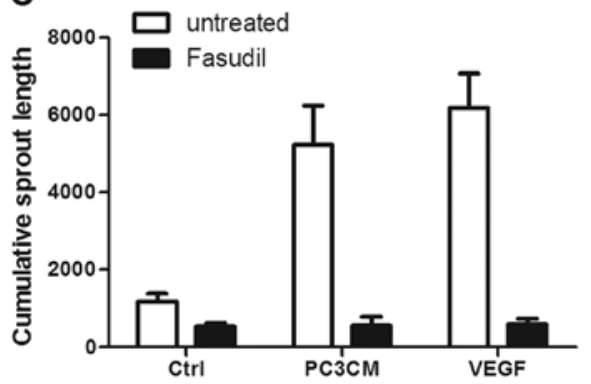

D

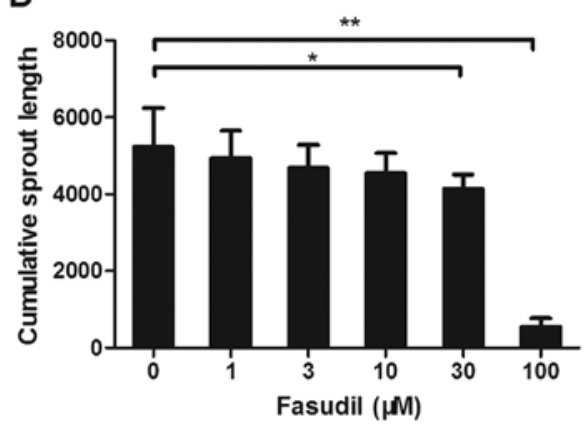

Figure 4. PC3CM-induced HUVEC spheroid sprouting is decreased by fasudil. (A) The sprouting of capillary-like structures from collagen-embedded HUVEC spheroids treated with fasudil $(100 \mu \mathrm{M})$ or left untreated was determined in basal medium (Ctrl), PC $3 \mathrm{CM}$, or basal medium containing $30 \mathrm{ng} / \mathrm{ml}$ VEGF. After $16 \mathrm{~h}$, sprouting was digitally recorded using a phase-contrast microscope. Sprouting from HUVEC spheroids stimulated with PC3CM (b) was almost completely inhibited by fasudil (e). (B) Fasudil decreased HUVEC sprouting in a dose-dependent manner, and $100 \mu \mathrm{M}$ fasudil inhibited sprouting almost completely. With increasing concentration of fasudil from $10-30 \mu \mathrm{M}$, the sprouts became thinner and more abundant compared with the ordered architecture of the untreated HUVEC spheroid sprouts. These sprouts were more like cell protrusions. (C and D) Quantification of sprouts from 10 spheroids (mean \pm SEM) was assessed by evaluating the cumulative sprout length per spheroid, and data were verified by three independent experiments. ${ }^{*} \mathrm{P}<0.05$; ${ }^{* *} \mathrm{P}<0.01$. HUVECs, human umbilical vein endothelial cells; PCa, prostate cancer; PC3CM, PCa cell line PC3-conditioned media; SEM, standard error of the mean; VEGF, vascular endothelial growth factor.

assay. When seeded onto Matrigel, HUVECs form tube structures and connect with each other, mimicking the in vivo process of angiogenesis. Sixteen hours after seeding, untreated HUVECs exhibited a clear capillary-like network forma- tion. However, fasudil treatment dramatically decreased the capillary-like network formation in a dose-dependent manner. As fasudil concentration increased, total tube length gradually decreased (Fig. 3). 

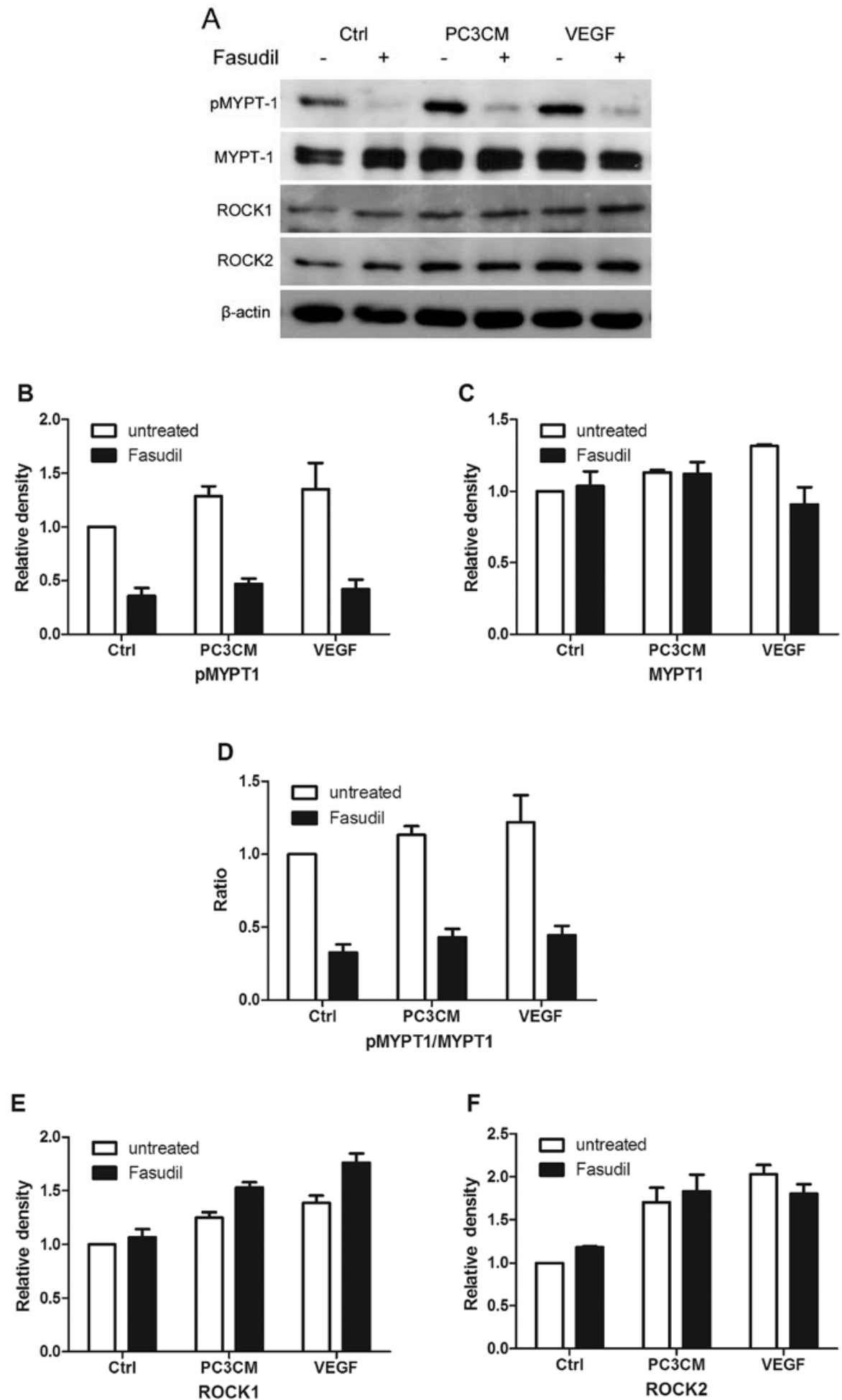

Figure 5. Fasudil inhibits PC3CM-induced ROCK activity. HUVECs were cultured in either basal medium (Ctrl), PC3CM, or basal medium containing VEGF $(30 \mathrm{ng} / \mathrm{ml}$ ) and treated with $30 \mu \mathrm{M}$ fasudil or left untreated. ROCK expression was detected by immunoblotting with anti-ROCK1 and anti-ROCK2 antibodies. ROCK activity was detected by phosphorylation of the downstream effector, MYPT-1, with anti-phospho-MYPT-1 and anti-MYPT-1 antibodies. (A) Representative images of western blots are shown. The relative density of each blot was quantified as fold-expression relative to the control. The data shown are the mean \pm SEM of 6 independent experiments. PC3CM increased pMYPT-1 expression and fasudil decreased pMYPT-1 expression (B) without any significant changes in MYPT-1 (C), resulting in an increase in the pMYPT-1/MYPT-1 ratio (D) in the PC3CM group and a decrease in this ratio after fasudil treatment. (E and F) PC3CM increased ROCK1 and ROCK2 expression while fasudil had no effect on ROCK expression. HUVECs, human umbilical vein endothelial cell; MYPT, myosin phosphatase target subunit 1; PCa, prostate cancer; PC3CM, PCa cell line PC3-conditioned media; ROCK, Rho-associated protein kinase; SEM, standard error of the mean; VEGF, vascular endothelial growth factor.

Fasudil inhibits PC3CM-induced HUVEC spheroid sprouting. In the sprout formation assay, HUVECs seeded in non-adhesive conditions in round bottom 96-well plates contributed to the formation of a single spheroid with a quiescent, non-proliferating surface monolayer within $24 \mathrm{~h}$. The spheroids were then embedded in a 3D collagen matrix. 
In the untreated control group, baseline sprouting was low (Fig. 4Aa). When cultured with PC3CM (Fig. 4Ab), baseline sprouting increased dramatically, although it was still less than that in the cells cultured with basal medium containing $30 \mathrm{ng} / \mathrm{ml}$ VEGF (Fig. 4Ac). Sprouting was almost completely inhibited by treatment with $100 \mu \mathrm{M}$ fasudil (Fig. 4Ad-f). We then examined the dose-dependent response of fasudil on PCa-induced HUVEC sprouting. As shown in Fig. 4B, fasudil decreased HUVEC sprouting in a dose-dependent manner and $100 \mu \mathrm{M}$ fasudil again inhibited sprouting almost completely.

Furthermore, when treated with increasing concentrations of fasudil, the sprouts became thinner and the HUVEC nucleus seldom emerged from the spheroids. These sprouts resembled cell protrusions, were markedly thinner compared with the untreated HUVEC sprouts, and were more abundant compared with the ordered architecture of the single HUVEC spheroid sprouts (Fig. 4B).

Fasudil inhibits PC3CM-induced HUVEC ROCK activation. MYPT-1 is one of the most crucial downstream effectors of ROCK. As fasudil is a ROCK inhibitor, we examined the inhibitory effects of fasudil on ROCK by measuring phosphoMYPT-1 (pMYPT-1), the active form of MYPT-1.

As shown in Fig. 5, when cultured with PC3CM or basal medium containing VEGF, expression of both MYPT-1 and pMYPT-1 was increased in the HUVECs, resulting in a moderate increase in the pMYPT-1/MYPT-1 ratio, indicating ROCK activation. Fasudil treatment lead to a significant decrease in pMYPT-1 and a slight decrease in MYPT-1, resulting in a significant decrease in the pMYPT-1/MYPT-1 ratio (Fig. 5A-D). A moderate increase in ROCK1 and ROCK2 expression was also detected, but ROCK expression was not altered significantly by fasudil treatment (Fig. 5E and F).

\section{Discussion}

To our knowledge, there have been no previous reports on the effects of fasudil on PCa-induced angiogenesis. In this study, HUVECs were cultured with the PCa cell line PC3CM to mimic endothelial cells in PCa tissue. Fasudil was then added to examine its effects on PC3CM-induced HUVECs using in vitro angiogenesis assays.

When cultured with PC3CM, ROCK1 and ROCK2 expression increased in the HUVECs, as did pMYPT-1 and total MYPT-1 expression. The pMYPT-1/MYPT-1 ratio was also increased. This indicates activation of the RhoA/ROCK pathway in PC3CM-stimulated HUVECs. It has been reported that endothelial cells in PCa tissue from TRAMP mice, a spontaneous PCa mouse model, have a constitutively high baseline level of activity of Rho GTPase and its downstream effector ROCK (17). This suggests that the RhoA/ROCK pathway plays a crucial role in $\mathrm{PCa}$ angiogenesis. HUVECs cultured in PC3CM share some of the characteristics of $\mathrm{PCa}$ endothelium and can therefore be used to represent it.

Angiogenesis involves a complex series of events that take place in a multi-step process. Endothelial cells migrate through the basement membrane toward an angiogenic stimulus. The leading front of migrating cells is driven by enhanced proliferation of endothelial cells, followed by the formation of capillary tubes via endothelial cell organization. The RhoA/ROCK pathway plays a role in each of these steps.

We evaluated the effects of fasudil on each of these steps in PCa-induced HUVECs. Fasudil was found to inhibit PC3CM-induced HUVEC proliferation, migration, tube formation and spheroid sprouting. This is in accordance with previous studies on VEGF-induced endothelial cell proliferation, migration and tube formation after treatment with the RhoA inhibitor, C3, or ROCK inhibitors, Y-27632 and fasudil $(15,16,18)$.

It is interesting to note the morphological changes that occurred in the spheroid sprouting assay after treatment with fasudil. After treatment with $10 \mu \mathrm{M}$ fasudil, the sprouts were much thinner than those on untreated cells. However, the HUVEC nucleus was observed less frequently moving out of the spheroids than in the controls. The movement of the nucleus decreased as the fasudil concentration increased, whereas sprouting was not affected until the concentration of fasudil exceeded $30 \mu \mathrm{M}$. These sprouts were more akin to cell protrusions, were markedly thinner compared with PC3CM-induced HUVEC sprouts, and were more abundant and disorganized compared with the ordered architecture of single HUVEC spheroid sprouting.

In conclusion, fasudil significantly inhibits the key steps of endothelial cell angiogenesis, including proliferation, migration and capillary tube formation, in a dose-dependent manner. These effects may be due to inhibition of ROCK activity induced by PCa cell secretions. Fasudil may be a useful antiangiogenic agent and should be investigated further for its potential role in the anti-angiogenic treatment of PCa.

\section{Acknowledgements}

This study was supported by a grant from The Program of International Science and Technology Cooperation (grant no. 2012DFG31440), awarded by The Ministry of Science and Technology, P.R. China. The authors are grateful to NewMed Publishing Services for providing final editing services.

\section{References}

1. Bubendorf L, Schöpfer A, Wagner U, et al: Metastatic patterns of prostate cancer: An autopsy study of 1,589 patients. Hum Pathol 31: 578-583, 2000.

2. Roehl KA, Han M, Ramos CG, Antenor JA and Catalona WJ: Cancer progression and survival rates following anatomical radical retropubic prostatectomy in 3,478 consecutive patients: long-term results. J Urol 172: 910-914, 2004.

3. Hull GW, Rabbani F, Abbas F, Wheeler TM, Kattan MW and Scardino PT: Cancer control with radical prostatectomy alone in 1,000 consecutive patients. J Urol 167: 528-534, 2002.

4. Daneshgari F and Crawford ED: Endocrine therapy of advanced carcinoma of the prostate. Cancer 71: 1089-1097, 1993.

5. Eymard JC, Oudard S, Gravis G, et al: Docetaxel reintroduction in patients with metastatic castration-resistant docetaxel-sensitive prostate cancer: a retrospective multicentre study. BJU Int 106: 974-978, 2010.

6. Bigler SA, Deering RE and Brawer MK: Comparison of microscopic vascularity in benign and malignant prostate tissue. Hum Pathol 24: 220-226, 1993.

7. Stefanou D, Batistatou A, Kamina S, Arkoumani E, Papachristou DJ and Agnantis NJ: Expression of vascular endothelial growth factor (VEGF) and association with microvessel density in benign prostatic hyperplasia and prostate cancer. In Vivo 18: 155-160, 2004. 
8. Weidner N, Carroll PR, Flax J, Blumenfeld W and Folkman J: Tumor angiogenesis correlates with metastasis in invasive prostate carcinoma. Am J Pathol 143: 401-409, 1993.

9. Borre M, Offersen BV, Nerstrom B and Overgaard J: Microvessel density predicts survival in prostate cancer patients subjected to watchful waiting. Br J Cancer 78: 940-944, 1998.

10. Green MM, Hiley CT, Shanks JH, et al: Expression of vascular endothelial growth factor (VEGF) in locally invasive prostate cancer is prognostic for radiotherapy outcome. Int $\mathrm{J}$ Radiat Oncol Biol Phys 67: 84-90, 2007.

11. Bok RA, Halabi S, Fei DT, et al: Vascular endothelial growth factor and basic fibroblast growth factor urine levels as predictors of outcome in hormone-refractory prostate cancer patients: a cancer and leukemia group B study. Cancer Res 61: 2533-2536, 2001.

12. Peyromaure M, Camparo P, Badoual C, Descazeaud A and Dinh-Xuan AT: The expression of vascular endothelial growth factor is associated with the risk of cancer progression after radical prostatectomy. BJU Int 99: 1150-1153, 2007.

13. George DJ, Halabi S, Shepard TF, et al: Prognostic significance of plasma vascular endothelial growth factor levels in patients with hormone-refractory prostate cancer treated on Cancer and Leukemia Group B 9480. Clin Cancer Res 7: 1932-1936, 2001.
14. Etienne-Manneville $S$ and Hall A: Rho GTPases in cell biology. Nature 420: 629-635, 2002.

15. van Nieuw Amerongen GP, Koolwijk P, Versteilen A and van Hinsbergh VW: Involvement of RhoA/Rho kinase signaling in VEGF-induced endothelial cell migration and angiogenesis in vitro. Arterioscler Thromb Vasc Biol 23: 211-217, 2002.

16. Yin L, Morishige K, Takahashi T, et al: Fasudil inhibits vascular endothelial growth factor-induced angiogenesis in vitro and in vivo. Mol Cancer Ther 6: 1517-1525, 2007.

17. Ghosh K, Thodeti CK, Dudley AC, Mammoto A, Klagsbrun M and Ingber DE: Tumor-derived endothelial cells exhibit aberrant Rho-mediated mechanosensing and abnormal angiogenesis in vitro. Proc Natl Acad Sci USA 105: 11305-11310, 2008.

18. Bryan BA, Dennstedt E, Mitchell DC, et al: RhoA/ROCK signaling is essential for multiple aspects of VEGF-mediated angiogenesis. FASEB J 24: 3186-3195, 2010. 